\title{
Violence politique, attentats et kamikazat : l'hypothèque du 11 septembre
}

\section{Daniel Hermant}

\section{(2) OpenEdition}

\section{Journals}

Édition électronique

URL : http://journals.openedition.org/conflits/2105

DOI : 10.4000/conflits.2105

ISSN : 1777-5345

Éditeur :

CCLS - Centre d'études sur les conflits lilberté et sécurité, L'Harmattan

\section{Édition imprimée}

Date de publication : 1 novembre 2006

Pagination : 13-23

ISBN : 2-296-01436-4

ISSN : 1157-996X

\section{Référence électronique}

Daniel Hermant, «Violence politique, attentats et kamikazat : I'hypothèque du 11 septembre », Cultures \& Conflits [En ligne], 63 | automne 2006, mis en ligne le 01 décembre 2006, consulté le 30 mars 2021. URL : http://journals.openedition.org/conflits/2105; DOI : https://doi.org/10.4000/conflits.2105

Ce document a été généré automatiquement le 30 mars 2021.

Creative Commons License 


\title{
Violence politique, attentats et kamikazat : l'hypothèque du 11 septembre
}

\author{
Daniel Hermant
}

«Un fait mal interprété par une époque reste un fait pour l'historien. C'est au gré

de l'épistémologue, un obstacle, c'est une contre-pensée ${ }^{1}{ }^{1}$.

1 Ce numéro, même si bien sûr il ne se limite pas à cela, a trouvé l'une de ses origines dans l'attentat princeps que fut le 11 septembre, tant la focalisation terrifiée sur le modus operandi de ce drame a contribué à mettre au premier plan la notion d'attentatsuicide. Ensuite, et notamment à travers l'usage débridé du mot «kamikaze ", s'est imposée une sorte de reprofilage de la notion d'attentat terroriste.

2 Certes, on ne saurait réduire sans abus à un attentat, aussi considérable soit-il, la question de la mort volontaire dans un contexte belliqueux, question qui, rappelons-le, traverse l'histoire, de l'Antiquité à nos jours et qui a revêtu de nombreuses formes que ce numéro analyse. De surcroît, et surtout dans cette revue, il ne saurait être question de faire du 11 septembre la mutation fondamentale, voire le commencement absolu que beaucoup y ont vu; cependant il ne faudrait pas a contrario raboter toutes les aspérités de l'événement et n'y voir que du spectaculaire, dont l'inusabilité proviendrait pour l'essentiel d'un discours de peur. Tout se passe en effet comme si, avec le 11 septembre, pour le plus grand nombre, mais également pour le petit monde des experts et des spécialistes, une nouvelle conception de l'attentat terroriste se mettait en place autour de la matrice implicite du kamikazat. A notre sens, il existe une véritable hypothèque du 11 septembre qui pèse sur les esprits et qui rend difficile tout examen objectif de la question des attentats-suicides. C'est cette hypothèque que nous voudrions examiner.

Retournement

3 Les attentats-suicides modifient notre perception de la violence et des conflits en en redistribuant les éléments constitutifs, et nous donnent ainsi l'impression d'une modification considérable du paysage. La focalisation du regard sur l'exécutant, plus que sur les dimensions collectives idéologiques politiques ou logistiques de l'action, la 
primauté de l'événement sur les mécanismes organisationnels, occultent pour l'opinion tout processus d'élaboration, toute friction avec le réel, toute atténuation par la force des choses. Au long terme d'un attentat préparé par des organisations et des planificateurs, succède la rapidité d'un événement conditionné par un choix individuel, voire d'un réflexe pathologique; au calcul stratégique des gains et des pertes succède l'anomie d'une violence extrême, à la raison politique, la passion ou le ressentiment, à la pondération, l'improvisation. L'organisation, l'Etat, la logistique, les forces collectives et, à un moindre degré, les cibles, bref tout ce qui constitue pour l'observateur le palpable de la violence - et qui ossifie son jugement -, perd sa position prééminente dans l'analyse et ne vient désormais qu'en second lieu. La perception des rapports de forces ne peut se trouver que bouleversée dans cette hypothèse, puisque nous passons d'un monde où les événements, en quelque sorte nourris par les réalités, étaient le reflet de leur enracinement, où l'action était liée par son contexte, à un monde de rapports de craintes dans lequel le calcul stratégique s'efface devant une logique de coups, c'est-à-dire d'événements qui se succèdent de manière aléatoire plus qu'ils ne s'enchaînent. Le monde apparaît fait de retournements ou de ruptures. Certes la guerre clausewitzienne n'est pas morte, mais dans l'opinion, ce sont ses cadres stables d'analyse qui ont perdu leur position centrale.

Radicalité

4 Ainsi, avec l'attentat-suicide l'individu, rouage central de la violence, promu human bomber, retrouve une sorte de prééminence, et l'analyse psychologique reprend toute sa place dans nos réactions. La perception de la violence se fait à l'aune de ce qu'il y a de plus intime et souvent de plus volatil en nous, la volonté individuelle.

5 Celle-ci apparaît d'abord dans l'idéologie des attentats-suicides. Comme il est difficile d'interroger un kamikaze sur ses intentions, surtout après son geste, celles-ci n'apparaissent aux sociétés cibles - les nôtres - qu'à travers la mise en scène et la propagande des discours de revendications, des bandes vidéos ou des photos " immortalisant» les kamikazes ou les meurtres d'otages. "Le mort saisit le vif", disent les juristes, ici c'est l'inverse, les morts - tous les morts: otages, kamikazes, victimes anonymes - sont instrumentalisés par les commanditaires. Il y a là pour la presse, comme pour le quidam, un effet de loupe et un message effrayant de violence, de sauvagerie, que la personnalité de Zarkaoui a assez bien illustré. Nous sommes la cible, et nous voici ramenés au statut de bétail qu'on égorge. A travers ces imprécations ou ces séquences sanglantes - qui choqueront jusqu'à ceux qu'elles sont censées servir - les valeurs de l'ennemi, c'est-à-dire les nôtres, refus de la mort, protection de la vie humaine, respect des droits de l'Homme, sont présentées comme une preuve de faiblesse, voire de couardise et leur inverse comme la promesse du triomphe. On ne sera pas surpris que, dans nos esprits, la radicalité de ce comportement, rende le kamikazat indissociable du fanatisme.

6 Mais il existe également une dimension autocentrée de ce radicalisme, celle qu'on tente de déchiffrer, au-delà de la propagande, à travers le mystère d'un visage qui annonce tranquillement sa mort pour demain. Le suicide politique présente un profil qui interpelle et fascine, il y a incontestablement du chemin de Damas dans cette décisionlà. Figure de la rupture, le kamikaze relève d'un choix radical, car comment être plus radical qu'en s'immolant?

7 Quand on se penche sur les trajectoires individuelles, ce qui inquiète, c'est qu'il ne s'agit pas d'un complot ourdi par de puissantes organisations transnationales contre 
lesquelles la puissance publique a les moyens de se défendre, mais à partir de l'air du temps, d'un basculement de vie, sociologiquement et statistiquement non significatif aléatoire donc -, mais à l'égard duquel on se sent désarmé. Autant dire qu'on ne sait pas grand-chose sur un processus de radicalisation politique où le passage à la violence résulte de micro-décisions individuelles, bien souvent inaperçues, au moins dans un premier temps, par l'entourage proche, mais qui vont entraîner par la levée d'interdits majeurs comme le suicide ou la loyauté envers la société dans laquelle on vit, une sorte de situation de transgression non transgressive qui facilite le passage à l'acte.

Or depuis le 11 septembre, cette posture fait florès. L'idée répandue en Occident que la mort volontaire renvoyait à un archaïsme religieux, lui-même issu d'un blocage sociétal dans le monde arabo-musulman, était supportable, parce qu'elle nous plaçait sur le bon côté de l'évolution et condamnait à l'érosion ce fanatisme issu du passé. Celle selon laquelle les volontaires de la mort sont le produit d'un échec de la modernisation dans les pays arabo-musulmans, quoique désagréable, l'était également parce qu'elle ne nous concernait qu'indirectement. En revanche, l'idée que la mort volontaire soit le résultat $\mathrm{du}$ rejet de nos sociétés occidentales, par les populations hétérogènes qui s'y sont installées, ne l'est pas. Dans un premier temps, on a pu essayer de contourner la difficulté en interprétant ces trajectoires comme des évolutions individuelles vers un nouvel idéal retrouvant, cinquante ans après, mais à rebours, une sorte d'exotisme révolutionnaire à la Régis Debray. A Madrid (11 mars 2004), le fait de la proximité géographique - une cellule marocaine - et d'une immigration trop récente, pouvaient encore servir d'alibi à la thèse d'un terrorisme importé, exogène. A Londres ( 7 juillet 2005), il a bien fallu, après de nombreuses dénégations, convenir que le ver était dans le fruit, que les kamikazes étaient issus du cru et faisaient peu de cas de la loyauté à la société dans laquelle ils avaient été élevés.

9 Malgré la répulsion qu'il provoque, malgré les accusations d'archaïsme religieux qu'il véhicule, on était obligé de constater que chez nous, à son échelle, depuis le 11 septembre, le kamikazat était puissamment mimétique et que, du salafisme, de la délinquance ou de l'ennui, au djihadisme, le chemin était souvent court ${ }^{2}$. Ce jusqu'auboutisme des candidats à la mort effraie et, par résonance, installe dans les opinions et les polices - incapables de détecter le futur kamikaze, une atmosphère du soupçon faite d'anxiété qui empoisonne nos sociétés.

Escalade

10 A cette radicalité du comportement kamikaze s'ajoute une radicalité de l'attentat luimême. On a parlé d'hyper-terrorisme ou de terrorisme d'un troisième type à propos du 11 septembre et beaucoup ont signalé la nouvelle ère que cet attentat hors norme inaugurait ${ }^{3}$. Question de bon sens, le recours au suicide est perçu comme retournant d'une manière spectaculaire le principe de Clausewitz selon lequel l'attaque est toujours plus dispendieuse que la défense puisque, par l'accès aux armes de destruction massives, quelques individus peuvent déstabiliser gravement nos sociétés, voire altérer ou bouleverser la donne stratégique mondiale. Le faible a trouvé la parade au fort. L'attentat-suicide est donc, autre formule qui traîne dans les médias, «la bombe atomique du pauvre »! L'affaire de l'anthrax aux Etats-Unis après le 11 septembre, la recherche de laboratoires chimiques en Afghanistan, le souvenir de l'attentat au gaz sarin dans le métro de Tokyo, ou la récente révélation d'un projet d'empoisonnement, toujours dans le métro, à New York sont les brouillons de ce qui pourrait être en préparation et concrétisent dans nos esprits l'idée que la triade des armes de 
destruction massive dites NBC est, ou va être, dans la besace des kamikazes. Le 11 septembre, qui dans nos esprits par sa dimension spectaculaire agrège tout ces éléments, se constitue en matrice symbolique de l'escalade. Certes, rien de cela ne s'est passé, mais l'actualité nous ramène toujours à cette perspective, et les simulations d'attentats réalisées un peu partout par les autorités ne sont pas de simples manipulations. On comprend le lien congénital du kamikazat avec les armes de destruction massive. Depuis le 11 septembre, dans les esprits, les attentats-suicides par leur rapport quasi naturel avec l'escalade sont tendanciellement de petites apocalypses. Indignation

11 L'onde de choc produite dans la société cible, et dans le monde entier pour le 11 septembre, par les attentats kamikazes, sature l'espace collectif, individuel, médiatique et bien sûr politique. Elle provoque un réflexe de cohésion autour des pouvoirs publics, renforce les solidarités et produit des héros : pompiers de New York, anonymes du vol 93, vidéos caméras de Londres. La pitié devant la souffrance et la solidarité dans le drame se transforment quasi immédiatement en indignation et en colère, car ces morts sont des victimes. Contre les coupables, l'unanimité est requise, la tiédeur, l'indifférence sont suspectes, aucune demie mesure, aucun demi sentiment n'est autorisé. Cette situation définie comme une guerre, au sens réel du terme dans Afghanistan des Talibans, ou avec une forte dimension métaphorique dans la "guerre au terrorisme", fonctionne sur le registre de l'émotion. La colère et l'indignation passionnée des populations visées traitent l'intention de nuire, le terme est faible, comme indivise, et considèrent ses auteurs, coauteurs ou sympathisants, comme coupables. L'émotion prend toute sa dimension parce que, au-delà du moment de l'attentat, elle se projette dans les projets inquiétants des commanditaires, et au-delà de la violence "physique», elle s'enracine dans l'opposition des valeurs: victime/ bourreau, légitime/illégitime, bon-bien/mauvais-mal. Parler des attentats kamikazes, ce n'est pas expliquer, c'est réprouver; ce n'est pas dénoncer, c'est prévenir. Nous sommes en face d'une véritable topique de l'indignation qui élimine du débat les tiers, ou plutôt qui les annexe ${ }^{4}$. Pourtant, dès que l'attentat s'éloigne, que la tension baisse, cette topique rencontre son envers. Les manières, le comment, le combien, le qui rendent floue l'imputation, multiplient les éléments de contexte, c'est-à-dire les circonstances atténuantes, bref, estompent la bifurcation vertigineuse des valeurs et ouvre un espace de discussions infinies sur la nature du terrorisme : quoi Al-Qaïda ? Qui Al-Qaïda? Pourquoi Al-Qaïda? Et plus encore de polémiques sur l'efficacité des mesures anti-terroristes dont il est si facile de montrer les contraintes et si difficile de mesurer l'efficacité. La lucidité analytique est rétrospective, la prise en compte de la menace prospective.

Temporalité

12 Ainsi prendre la mesure de ces attentats, ce n'est pas décrypter ce qui est échu, mais supputer ce qui peut arriver. Or tout peut arriver, car l'attentat est "expansif», il se répand dans les esprits où il a tendance à occuper tout l'espace disponible. On ne peut l'enfermer dans un cadre déductif simple, le ramener à la réalité de ce qui a eu lieu, car son référent principal n'est pas le passé mais l'avenir. François Hertog ${ }^{5}$, réfléchissant sur la temporalité, distinguait une posture classique où le passé éclairait le présent et dessinait l'avenir comme un retour à l'âge d'or, une posture futuriste où l'avenir radieux guidait le présent et bien souvent l'écrasait sous le poids des utopies, en gros celles du xixe siècle et du xxe siècle, et enfin la posture actuelle, celle d'un présent omnipotent qu'il baptisait «présentisme », néologisme laid mais éclairant. Le présent 
relit le passé en fonction des préoccupations du moment, pour ensuite réinjecter dans l'actualité ce qu'on pourrait appeler un passé recomposé ${ }^{6}$. Mais, autre dimension du présentisme, l'avenir change lui aussi de statut puisqu'il est en quelque sorte intégré au présent. Le rapport présent/futur n'est plus un rapport de succession, mais une sorte d'alliage immédiat, forgé par la puissance des moyens d'action à notre disposition, dont l'efficacité, outre le présent, asservit l'avenir ${ }^{7}$. Le principe de précaution, très en vogue chez les écologistes, sorte de coïtus interruptus sociétal qui tente de maîtriser par l'abstinence les effets potentiellement désastreux de la techno-science, illustre à merveille cette posture. On voit bien comment le kamikazat prend sa place dans ce schéma : l'horizon qui polarise tout le champ et qu'aucun gouvernement responsable ne peut exclure sans légèreté, est celui d'apocalypse to morrow. Certes, il ne s'agit que d'une hypothèse, mais elle est à considérer sérieusement car les kamikazes et leurs commanditaires en rêvent ${ }^{8}$. Ce surplomb insupportable de l'avenir sur notre présent peut, par réaction, entraîner certains observateurs à s'ancrer au maximum dans la réalité échue pour retrouver un terrain solide où les compétences de l'analyste peuvent se déployer. Il s'agit de remplacer les spéculations sur les auteurs des attentats et sur leurs intentions par l'examen des réactions des gouvernements, des services et des médias, parfaitement lisibles - et même pré-lisibles -, parce que déjà inscrites dans le fonctionnement des pouvoirs publics et de la société, et renvoyant à une histoire au long terme qui digère les événements au moins autant qu'elle ne les subit. On se trouve ainsi en face d'un contrepoint entre ce qu'on pourrait appeler d'une part une heuristique de la peur ${ }^{9}$, et d'autre part une heuristique de la prudence. La première, inscrite dans la rupture du 11 septembre, a pour objet en tenant comme probable, et même comme très probable, la possibilité d'une surenchère «ben ladiniste » de nature biologique ou chimique, de tenter de la rendre improbable, sinon impossible, par la mise en œuvre de politiques sécuritaires. La seconde propose une interprétation minimaliste des attentats, mais transfère ses craintes sur les effets potentiellement liberticides des politiques anti-terroristes. Au cœur de ce malentendu se trouve l'idée d'une relation sécurité/liberté structurellement contradictoire, chaque avancée de la sécurité ne pouvant que renvoyer à un recul du droit et de la liberté, alors que ces deux notions sont complémentaires et doivent être mises en tension. Leur face-à-face étroit, asphyxiant, qui se rétablit à chaque crise devant l'opinion, nous égare dans un espace polémique.

Brouillages

13 Ces deux manières de réagir à ce véritable tremblement de temps qu'est le 11 septembre brouillent le débat en lui donnant une tonalité fortement idéologique car, comme cela arrive souvent, elles transforment leurs objections en réalités, et leurs critiques en lois! On peut ainsi dénoncer ceux qui voient dans le 11 septembre - et ils sont nombreux - la coupure décisive entre l'ancien temps/monde et le nouveau pour, à l'inverse, normaliser l'événement, en raboter les aspérités en quantifiant et en chronologisant comme s'il s'agissait d'une simple série ${ }^{10}$. On peut, dans le même esprit, montrer l'irrationnel des réactions à chaud, ironiser sur les dérapages verbaux et la mobilisation émotionnelle, souligner l'égarement du moment et les effets de l'incertitude et de l'escalade sur la peur ou le désir de vengeance. Mais, en ramenant le 11 septembre à un manque de sang froid, à une exagération psychologique, voire à une réaction hyper-nationaliste et xénophobe, en le réduisant à une infraction de plus contre la sécurité de l'Etat, on s'expose à ne pas voir qu'il s'agit d'un crime de masse frappant des populations civiles et entraînant une dépersonnalisation des victimes. 
Cela l'apparente à un crime contre l'humanité, ce qui non seulement justifie le sentiment de rupture chronologique qu'on ressent, mais impose une réflexion sur le fond qui n'a pas encore été menée ${ }^{11}$. On peut pourtant aller plus loin encore dans cette sous-estimation, structuraliser en quelque sorte les causes du 11 septembre en en faisant le produit de l'arrogance occidentale, et plus spécifiquement américaine, au Moyen-Orient, une espèce de retour de boomerang, le résultat du passé plus que la menace sur l'avenir ${ }^{12}$. La dimension mimétique des attentats sur laquelle nous avons jadis insisté ${ }^{13}$ supposait l'existence de deux pôles distincts, elle est remplacée par une analyse moniste dans laquelle il n'y a plus qu'un seul acteur: l'Etat. Mais cette "dévitalisation" des terroristes entraîne-t-elle vraiment la conviction? Ben Laden, Zawahiri, Zarkaoui, Khaled Cheikh Mohamed ne sont-ils que des phénomènes dérivés, des effets pervers issus des manipulations de la CIA ? Peut-on sérieusement dire que le danger principal vienne moins des attentats eux-mêmes que des réactions disproportionnées de nos sociétés et de nos gouvernants qui, en amont, les provoquent, ou n'est-ce pas aller trop loin et s'exposer, par une sorte de retournement des causalités, à une exagération inverse de celle contre laquelle l'heuristique de la prudence s'était bâtie à juste titre ? Certes, il y a incontestablement une amplification psychologique de l'attentat kamikaze et une propension à l'escalade dans la réponse, car l'anti-terrorisme est autant un champ d'exagération qu'un champ d'action, et ce n'est pas être anti-américain que de dire que, dans cette voie, l'équipe du président G.W. Bush a beaucoup donné. De surcroît, ce mécanisme très puissant donne par un effet pervers une substance médiatique aux terroristes : c'est dans ce sens, et dans ce sens seulement, que Zarkaoui a pu être considéré en Irak comme une créature du Pentagone. En revanche, ces déformations d'images n'ont que peu à voir avec le danger réel présenté par les kamikazes ou leurs inspirateurs, et ne les transforme d'aucune manière en tigres de papier ou en artefacts informatiques.

Fausses semblances

14 On le voit, il y a, dans ces jeux d'interprétations, des glissements, voire des retournements de sens, qui nous obligent à la prudence. On pourrait parler de tête-àtête étouffant, quand chacun agite l'épouvantail de la figure adverse, de torsion du sens, tant on s'acharne à faire varier les arguments dans des directions opposées ; ainsi la comparaison entre G.W. Bush et Ben Laden souvent faite, reste en dernier ressort, simple rhétorique, et Guantanamo, quoiqu'en disent ceux qui dénoncent la mise en place d'un état d'exception depuis le 11 septembre, n'est l'équivalent ni réel ni symbolique du 11 septembre. En revanche, c'en est certainement une conséquence mal maîtrisée, comme le montre l'avis de la Cour suprême américaine du 3 juillet 2006, qui déclare illégaux les tribunaux militaires de Guantanamo ${ }^{14}$. De la même manière, la réalité d'une menace interne ne justifie pas n'importe quelle mesure de contrôle de population ${ }^{15}$, et il faut être conscient que la critique souvent fondée dans ce domaine, des errements des pouvoirs publics, entraîne subrepticement une approbation déguisée des violences que les autorités voulaient justement combattre : méditons sur la dérive qu'il y aurait à métamorphoser tel ou tel pied nickelé ou illuminé de retour de Guantanamo en héros de la démocratie ${ }^{16}$, à légitimer ce faux-monnayage où chacun s'efforce de paraître un autre!

Résumons-nous. La politique anti-terroriste et le discours qui la critique, marchent d'un pas différent dans la même direction: les opinions, les mises en garde, voire les refus du second ont pour unique objet de rendre plus efficace la lutte et l'action, ou au moins d'éviter aux autorités de tomber dans des chausse-trappes juridiques. Pourtant 
quand la pédagogie rectificatrice se transforme en polémique, quand la critique devient accusation et même stigmatisation, et le refus d'une mesure : rejet de la personne qui l'a prise, alors les objectifs se brouillent et même s'inversent. Le recouvrement, même partiel de l'anti-terrorisme par ce qu'on peut appeler «l'anti-anti-terrorisme», non seulement témoigne de l'emprise d'un discours militant agressif visant à délégitimer l'action des autorités, c'est-à-dire correspondant, comme l'indique le doublement de la négation, à un retournement de polarités, mais, de surcroît, dessine en creux, à travers un processus de victimisation, un espace de légitimation des kamikazes et de leurs thuriféraires!

Focale

16 Nous avons, dans les pages qui précèdent, examiné la réception du 11 septembre en Occident, sans chercher, comme le fait ce numéro, et plus largement la littérature sur le sujet, à distinguer entre la perspective globalisante des nouveaux martyrs d'Al-Qaïda et la pratique du suicide dans les conflits saturés - à enjeux territoriaux: Palestine, Tchétchénie, Sri Lanka, etc. - non par myopie, mais parce que le 11 septembre, et c'est un de ses caractères les plus importants, modifie le rapport entre le singulier et le général et induit une tendance irrépressible à la généralité. Dès qu'on aborde la question, c'est à l'échelle de la société tout entière qu'on veut la résoudre, ce qui nous situe dans la pétition de principes, l'anathème ou la compétition de légitimité, mais nous éloigne du terrain, des terrains devrait-on dire? Ainsi nous n'avons pas distingué entre les formes de violence, entre le suicide altruiste de Yann Palach à Prague en janvier 1969, qui manifeste la puissance du droit contre la force et le kamikaze irakien, qui n'est plus qu'un instrument commode aux mains de milices. Depuis le 11 septembre, la mort volontaire sert d'index au nouveau continuum de violence qui s'est mis en place et dans lequel la décapitation emblématisée entre en résonance avec la martyropathie des bombes humaines.

17 Nous n'avons pas suivi non plus l'errance géographique des attentats: New York, Madrid, Londres mais également Bali, Djerba, Charm el Cheikh, Bagdad, celui plus fourni encore des tentatives qui ont échoué, ou celui des sanctuaires, Soudan, Afghanistan, Waziristan, Somalie à nouveau? Et des pseudo-sanctuaires, Irak avant 2003. Pas plus que la liste des causes défendues: Tchétchénie, Palestine, Irak... Cette ubiquité inquiétante d'un mouvement qui ne se laisse pas enfermer dans un espace mais les articule tous, même si c'est bien souvent a posteriori et de façon opportuniste ${ }^{17}$, exigerait sans aucun doute une analyse de la dimension organisationnelle du kamikazat ${ }^{18}$, mais d'une certaine façon, peu importe car l'hypothèque que fait peser le 11 septembre sur nos esprits ne varie pas en fonction de la réalité et s'articule aussi bien à l'idée d'un réseau puissant, qu'à celle d'une nébuleuse, ou d'une simple instance légitimante.

Tremblements

18 Toute réflexion sur la violence politique et le kamikazat doit commencer par lever l'obstacle épistémologique que constitue le 11 septembre. En effet, il introduit une sorte de béance rompant avec les logiques d'action politique qui nous servent à déchiffrer l'actualité. L'attentat-suicide est comme un brouilleur d'analyse; il efface les lignes simples, démultiplie les perspectives, et fait trembler la réflexion. On a l'impression d'un tour en arrière de la roue de l'histoire, d'une régression. Longtemps, on a cru que les mœurs se civilisaient plus ou moins rapidement, que la violence régressait, or avec le 11 septembre, la juridisation de la société, la multiplication des procédures de 
négociation, de médiation ou de réconciliation cèdent le pas à une violence brutale et indiscriminée. Le kamikaze prend à contre-pied un Occident qui avait peu à peu soustrait la mort au regard, et impose de manière presque obscène le retour de l'autosacrifice sur la scène publique. Certes, il y a une histoire occidentale du sacrifice, mais celui-ci a été "symbolisé ", "euphémisé ", on lui a ôté sa chair et son sang; sa réapparition au premier plan sous une forme agressive dans laquelle on fusionne la mort d'autrui avec la sienne, ne peut que nous choquer. Les «fous d'Allah" courtcircuitent l'histoire, marquent le retour à une époque qu'on croyait révolue, avec le triomphe de la passion sur la raison, de la religion sur la politique. Leur engagement individuel est singulier, il relève du basculement, de la conversion, les voilà born again, mais ce singulier est un singulier pluriel qui, au niveau collectif, illustre une solidarité mécanique, faite d'imitation, où l'individu est étouffé par le groupe. Dans le même esprit, l'histoire devient chaotique, l'attentat est aléatoire, il n'entre pas a priori dans une série, même s'il peut se répéter il remplace l'efficacité par l'impact, le calcul par l'émotion, il ne construit pas, il démolit. Depuis le 11 septembre, on est dans le domaine de la saillance situationnelle, pas dans celui de la stratégie.

Cette dernière formulation, encore traditionnelle, rend certes compte de l'événement, avec sa réalité, et même son hyper-réalité, sa tendance à l'excessif, la dimension de transgression qu'il comporte. En revanche, elle appréhende assez mal le tremblement de temps qui nous paraît caractériser, pour ses partisans, le kamikazat depuis le 11 septembre. Le rapport de causalité passé/présent, passage obligé de toute explication, est remplacé par une fusion qui transforme chaque attentat en indice de l'accomplissement des temps au sens quasi religieux du terme. C'est la conscience confuse de l'efficace de cette mentalité - elle fait basculer des vies individuelles -, du danger du déni, et du peu d'efficacité de la raison raisonnante pour lutter contre cette attraction, qui rend si angoissante l'hypothèque que le 11 septembre fait peser sur nos sociétés. Combattez cette dimension et le kamikazat ne sera plus, comme dans l'Irak actuel, qu'un moyen, un procédé tactique, il s'affaissera.

\section{NOTES}

1.. Bachelard G., La Formation de l'esprit scientifique, Paris, Vrin, 2004.

2.. L'étude des trajectoires qui mènent à l'engagement violent et au kamikazat repose sur des sources nombreuses, dont la plus célèbre est le texte de Muhammad Atta retrouvé par le FBI dans ses bagages. Les procès, comme celui de Moussaoui, ou ceux dans les pays respectifs des libérés de Guantanamo ainsi que les enquêtes policières ou les rapports après les attentats de Londres ou de Madrid donnent également de nombreux renseignements. Voir Sageman M., Understanding Terror Networks, Philadelphia, University of Pennsylvania Press, 2004 ; et en français : Khosrokhavar F., Quand Al-Qaïda parle, Paris, Grasset, 2006.

3.. Voir par exemple pour son titre l'ouvrage collectif dirigé par Heisbourg F., Hyperterrorisme : la nouvelle guerre, Paris, Odile Jacob, 2001.

4.. Sur ce point, voir Boltanski L., La Souffrance à distance, Paris, Métailié, 1993. 
5.. Hartog F., Régimes d'historicité, présentisme et expérience du temps, Paris, Le Seuil, 2003. 6. Notre présent, décomposé à force de querelles mémorielles, en est la conséquence. 7.Dans le même sens, voir Beck U., La Société du risque. Sur la voie d'une autre modernité, Paris, Champ Flammarion, 2001. "Dans la société du risque, le passé perd sa fonction déterminante pour le présent. C'est l'avenir qui vient s'y substituer, et c'est alors quelque chose d'inexistant, de construit, de fictif, qui devient la "cause" de l'expérience et de l'action présente ", p. 61.

8. Pierre Hassner résume ce sentiment lors d'une table ronde tenue le 8 octobre 2001 : " Je suis persuadé - et la plupart des spécialistes aussi, même si c'est parfois avec quelques excès - qu'il y aura des attentats avec des armes chimiques ou biologiques, et peut-être un jour un avion ira se précipiter sur une centrale nucléaire avec des effets bien supérieurs à Tchernobyl. Qui dira encore à ce moment là : "rien de nouveau sous le soleil" ", " Table ronde : Le monde après le 11 septembre ", Critique internationale $\mathrm{n}^{\circ} 14$, op. cit., p. 62.

9.. Voir Dupuy J.-P., Pour un catastrophisme éclairé, Paris, Le Seuil, 2002, et Petite métaphysique des tsunamis, Paris, Le Seuil, 2005.

10.. Après tout, dit-on, le 11 septembre remonte maintenant à six ans et, depuis, pour rester dans la sphère occidentale - mais est-ce justifié ? - il n'y a eu que deux grands attentats : Madrid en 2004 et Londres en 2005, donc l'efficacité et la létalité sont en baisse.

11.. L'utilisation de la notion de " guerre contre le terrorisme » par les Etats-Unis, abondamment critiquée, montre bien la nécessité de définir avec précision les valeurs qui ont été transgressées par le 11 septembre, mais également les inconvénients qu'il y a à le faire sommairement.

12.. Cette attitude reprend sur un autre plan le discours même d'Al-Qaïda. Par exemple, « Les non-Musulmans méritent d'être attaqués parce qu'ils ont voté pour un gouvernement qui continue à opprimer nos mères, enfants, fils et sœurs en Afghanistan, Palestine, Irak et Tchétchénie ", affirme Shehzad Tanweer, auteur de l'explosion de la station de métro Aldgate à Londres.

13.. Bigo D., Hermant D., « La relation terroriste », Etudes polémologiques $n^{\circ} 47,1988$ et «Tiers, médiateurs et parasites », Etudes polémologiques $n^{\circ} 49,1989$.

14.. Avis dont bien entendu l'administration Bush tient compte, comme l'indique le mémorandum du 7 juillet 2006 du secrétaire adjoint à la Défense, Gordon England.

15.. Laisser la bride sur le cou aux services de sécurité qui, pour prévenir les attentats, multiplient les mesures de contrôle tous azimuts : vidéos de surveillance, ou profilage des groupes à risque, peut provoquer des excès qui finissent par insécuriser une partie de la population au moins autant qu'il ne sécurisent l'ensemble.

16.. Il y a en Occident, et tout particulièrement en France, une tradition intellectuelle qui octroie une sorte de prime à la critique de l'Etat et qui confère une espèce de légitimité à tout opposant.

17.. Les vidéos de Mohammad Sidique Khan et de Shehzad Tanweer diffusées par AlJazira le 1er septembre 2005 et le 6 juillet 2006 relèvent de cette logique, même si elles confirment le lien avec Al-Qaïda.

18.. Qui peut d'ailleurs être esquissée à travers les interprétations qui courent en Occident depuis 2001. 


\section{RÉSUMÉS}

Après le 11 septembre, autour du kamikazat, se met en place en Occident une nouvelle perception de l'attentat terroriste, dont l'auteur analyse les composantes : imprévisibilité, radicalité, tendance à l'escalade de la violence, tendance irrépressible à la généralisation, le tout hypothéquant l'avenir. Il montre ensuite les conséquences déstabilisantes de cette situation dans nos sociétés, notamment dans le domaine de l'anti-terrorisme devenu bien paradoxalement un sujet très polémique.

After September the 11th, the perception of terrorist-bombings has been restructured around the topic of suicide through the image of the kamikaze. The author analyses the underpinnings of this change that implies a focus on the unforeseeable character of violence, the tendency of radicalisation and that leads to inevitable generalisations. Because of its long-term consequences, this focus might amount to a "hijacking" of the future. He pursues by highlighting the destabilising consequences of this situation in our societies, in particular in the field of antiterrorism that paradoxically has become very polemic.

\section{INDEX}

Index chronologique : 2001 post 11 septembre

Mots-clés : attentat, kamikaze (missions suicide)

\section{AUTEUR}

\section{DANIEL HERMANT}

Ancien directeur de l'IFP, directeur de publication de Cultures \& Conflits, Daniel Hermant travaille sur la violence politique. 